\title{
Primary Chondrosarcoma of the Chest Wall- A Case Report
}

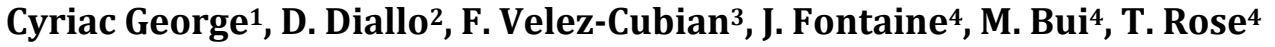 \\ ${ }^{1}$ Radiology Department, University of South Florida, Tampa, USA \\ ${ }^{2}$ General Surgical Department, University of South Florida, Tampa, USA \\ ${ }^{3}$ Thoracic Surgical Department, Moffitt Cancer Center, Tampa, USA \\ ${ }^{4}$ Pathology Department, Moffitt Cancer Center, Tampa, USA \\ Email: gcyriac@health.usf.edu
}

How to cite this paper: George, C., Diallo, D., Velez-Cubian, F., Fontaine, J., Bui, M. and Rose, T. (2018) Primary Chondrosarcoma of the Chest Wall-A Case Report. Open Journal of Radiology, 8, 150-158. https://doi.org/10.4236/ojrad.2018.83017

Received: July 14, 2018

Accepted: August 17, 2018

Published: August 20, 2018

Copyright $(9) 2018$ by authors and Scientific Research Publishing Inc. This work is licensed under the Creative Commons Attribution International License (CC BY 4.0).

http://creativecommons.org/licenses/by/4.0/

\begin{abstract}
A 60-year-old Hispanic male presented to his primary care physician office with an asymptomatic, but palpable right anterior chest wall mass. Initial work up of the finding included a CT scan of the chest which revealed a non-calcified, solid right anterior chest wall mass with invasion of the anterior fifth rib and intercostal space. The patient was presented at multidisciplinary conference with the patient's primary physician, a medical oncologist, radiologist, pathologist and oncologic surgeon in attendance. The decision was to perform surgical resection of the mass to treat this primary mesenchymal malignancy. The anterior aspect of the fifth rib and intercostal muscles were resected with negative margins. Pathology confirmed the mass to be a low-grade chondrosarcoma. Due to the low-grade nature, low metastatic potential and negative margins of the tumor, the decision was made not to pursue adjuvant chemotherapy or radiation therapy. The patient made full recovery.
\end{abstract}

\section{Keywords}

Chondrosarcoma, Chest Wall Tumors, Cartilaginous Tumors

\section{Introduction}

Chondrosarcomas are the most common primary chest wall malignancies and the second most common malignant bone tumors. The diagnosis and management warrant a multidisciplinary approach that includes the primary physician, medical oncologist, surgeon, pathologist and radiologist. Multi-modality imaging including CT, MRI and PET along with histopathological tissue analysis can 
be used to determine a conclusive diagnosis of chondrosarcoma.

\section{Case Presentation}

A 60-year-old Hispanic male presented to his primary care physician office with an asymptomatic, but palpable right anterior chest wall mass. The patient has no significant past medical history except for a clavicular fracture sustained during soccer. Physical examination revealed an asymptomatic $4 \mathrm{~cm}$ soft tissue palpable mass of the right anterior chest wall. The skin was intact, no erosive markings were present. The patient's laboratory work up was unremarkable. Radiology work up included CT chest, MRI of the chest and PET/CT. His CT chest axial series on bone windows demonstrated a $2.8 \times 3.8 \times 2.9 \mathrm{~cm}$ pleural based soft tissue mass eroding through the right anterior fifth rib (Figure 1). There was mild compression of the nearby lung parenchyma, however no pulmonary lesions were identified. Further characterization of the mass by MRI contrast enhanced images of the chest revealed a well-circumscribed pleural based T1 hypo-intense avidly enhancing soft tissue mass in the right anterior chest wall involving the right anterior fifth rib and into the overlying sub-pectoral soft tissues (Figure 2). The mass also demonstrated restricted diffusion which is indicative of high cellularity (Figure 3 and Figure 4). PET/CT imaging revealed mild hypermetabolic activity of the mass with maximum SUV of 1.9 (Figure 5).

The patient underwent a right-sided video-assisted thoracoscopy (VATS) with right chest wall resection and reconstruction with an advancement muscle flap. No synthetic material or plates were used. The patient tolerated the operation without complications and had an uneventful recovery.

Gross pathology of the fifth rib resection revealed a firm, oval, tan-white colored 3.7 LR $\times 3.3 \mathrm{CC} \times 3.2 \mathrm{AP} \mathrm{cm}$ solid mass (Figure 6). Cut surfaces of the mass were gray-white, focally mucinous and cartilaginous (Figure 7). A small

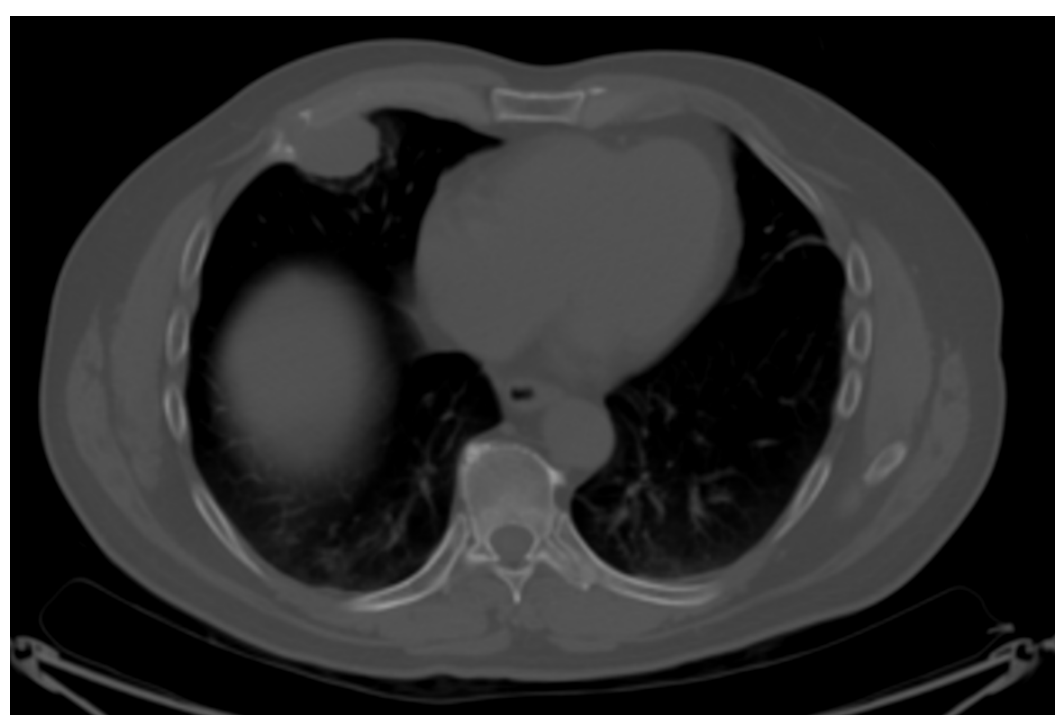

Figure 1. Axial CT of the chest using bone windows demonstrates a $2.8 \times 3.8 \times 2.9 \mathrm{~cm}$ soft tissue right chest wall mass involving the right anterior fifth rib. 

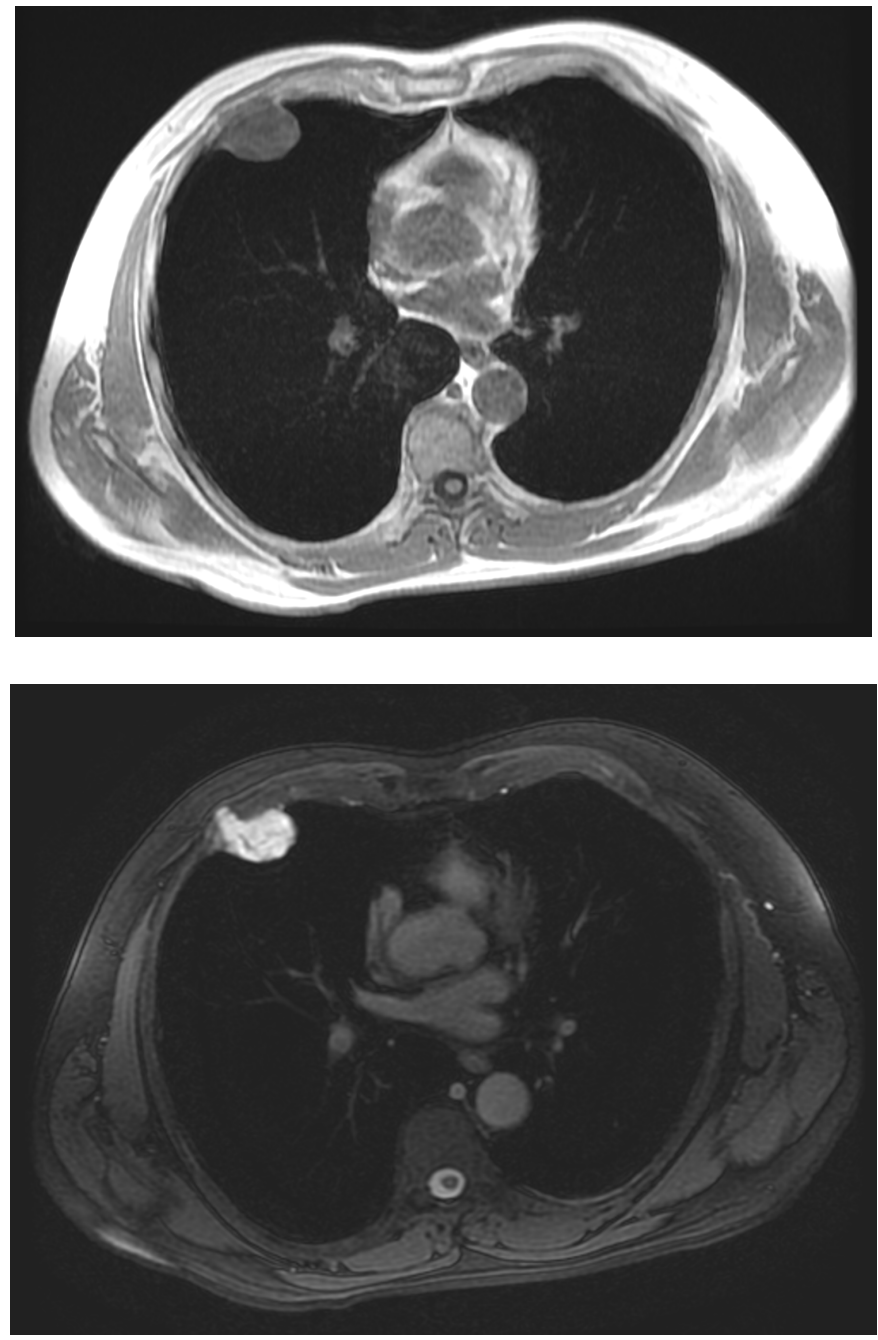

Figure 2. Axial post-contrast T1 weighted MRI image is noted for avid enhancement of the destructive anterior chest wall mass.

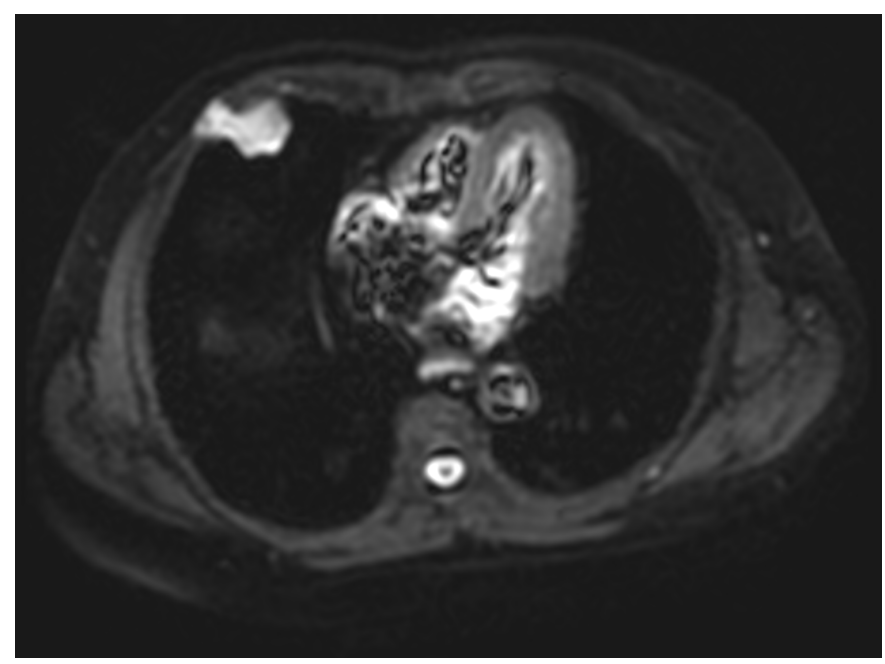

Figure 3. Diffusion weighted imaging (DWI) of the anterior chest wall mass shows hyperintense signal with corresponding hypointense signal on apparent diffusion imaging (ADC) shown in Figure 4, which indicates with the high cellularity of the tumor. 

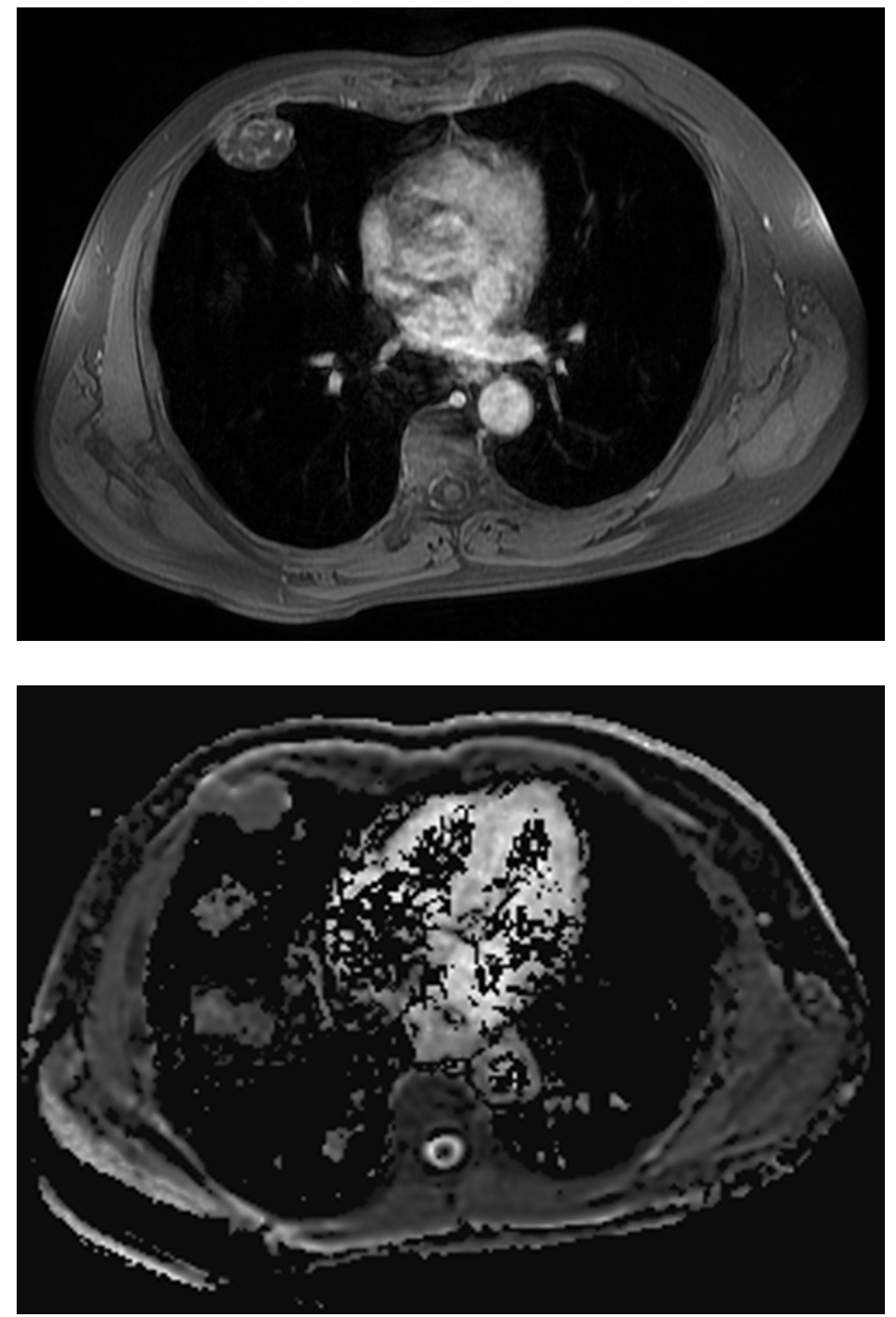

Figure 4. Apparent diffusion imaging correlate with hypointense signal of the mass.

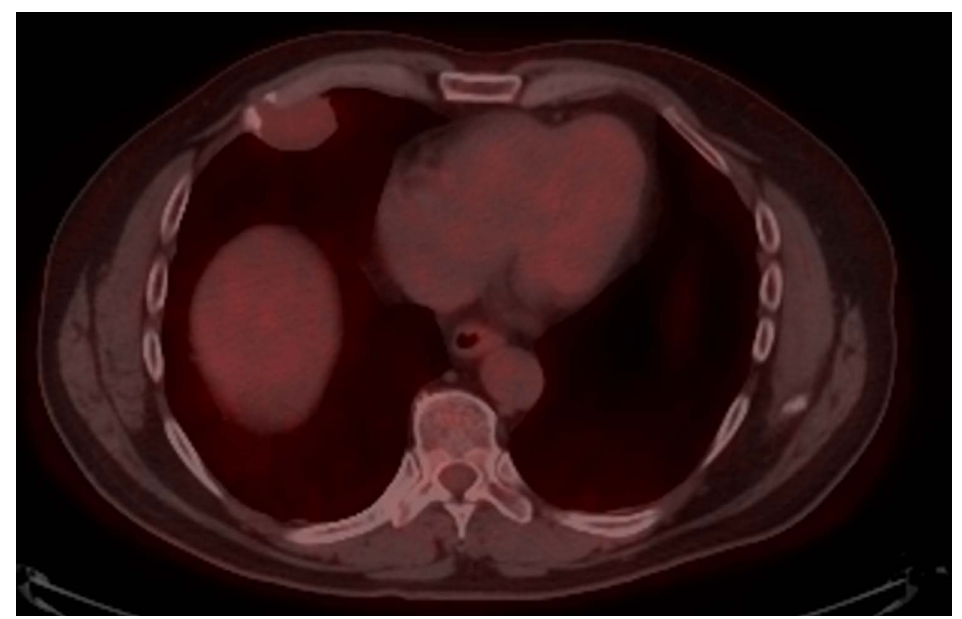

Figure 5. Axial fused PET/CT image at the level of the lower chest shows a soft tissue mass with osseous destruction of the anterior fifth rib with a maximum standard uptake value (SUV) of 1.9 . 


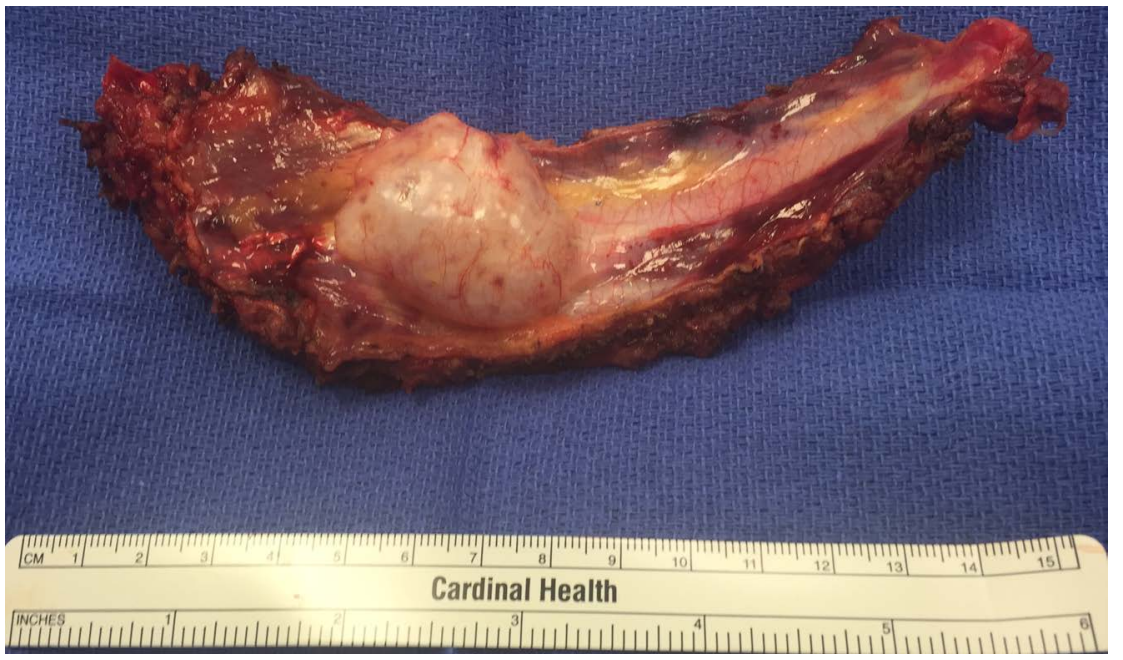

Figure 6. Gross pathology of the resected right fifth rib revealed a firm, oval, tan-white colored $3.7 \times 3.3 \times 3.2 \mathrm{~cm}$ solid mass.

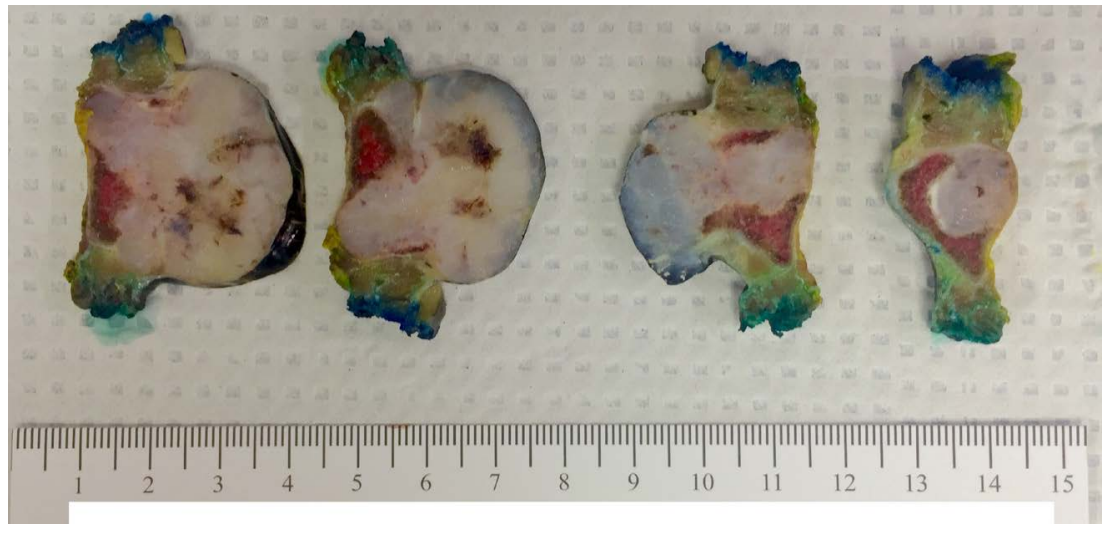

Figure 7. Cross sectional gross pathology shows the tumor invading the soft tissue arising from the benign cartilage.

portion of the mass was submitted for intraoperative frozen section diagnosis. The mass was serially sectioned from lateral to medial in $3-4 \mathrm{~mm}$ intervals into 9 slices. The mass grossly involved the bone and cartilage of the rib and abut the anterior and posterior surfaces. The mass measured $7 \mathrm{~mm}$ from the inferior soft tissue margin, $8 \mathrm{~mm}$ from the superior soft tissue margin, $5.6 \mathrm{~cm}$ from the lateral bone margin and $5.9 \mathrm{~cm}$ from the medial bone margin.

\section{Diagnosis}

In light of the presentation and imaging findings the following differential diagnoses were considered: peripheral nerve sheath tumor, plasmacytoma, lymphoma, enchondroma, osteosarcoma, chondrosarcoma, fibrous tumor of the pleura and metastatic disease. The laboratory work up was within normal limits eliminating lymphoma and plasmacytoma. Upon resection of the mass, the histopathology demonstrated benign cartilage which was consistent with Grade I chondrosarcoma (Figure 8 \& Figure 9). Due to the low-grade nature, low metastatic 


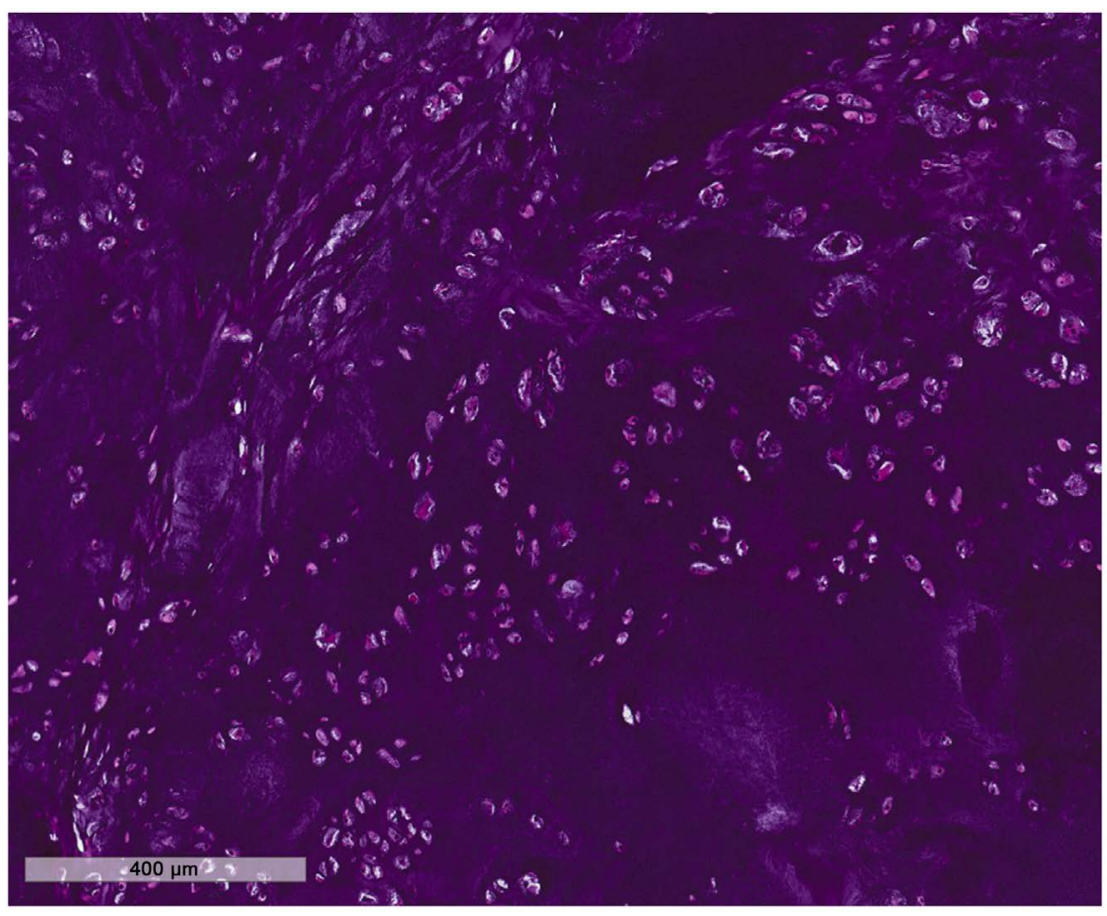

Figure 8. Micropathology slide low power magnification analysis was consistent with Grade I chondrosarcoma.

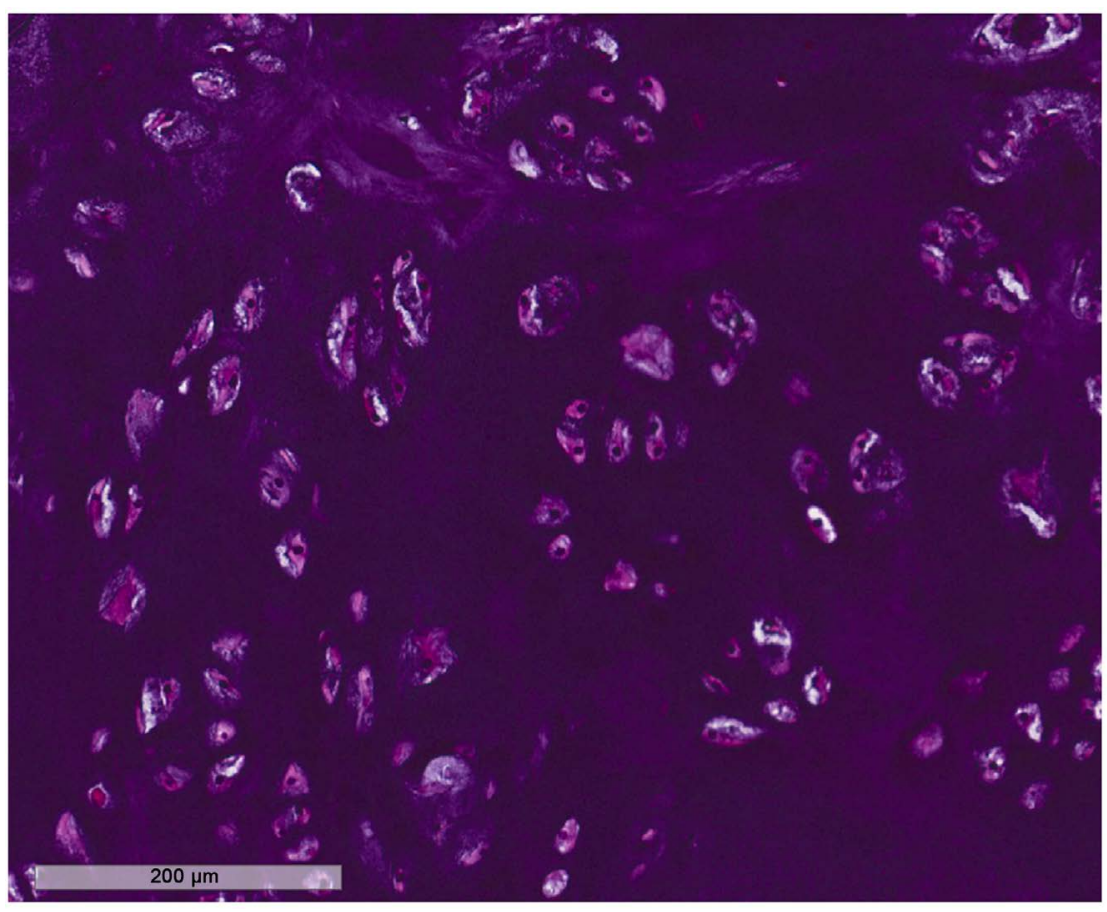

Figure 9. Micropathology slide high power magnification analysis was consistent with Grade I chondrosarcoma.

potential and negative margins of the tumor, the decision was made not to pursue adjuvant chemotherapy or radiation therapy. Patient gave consent for publication of this case report. At two years after surgical resection, patient remained 
negative for re-occurrence.

\section{Discussion}

\subsection{Clinical Findings}

Chondrosarcomas are the most common primary chest wall malignancies and the second most common malignant bone tumors. They are more commonly seen in males, usually presenting between the 4th-7th decades of life [1] [2] [3]. Patients can present with progressive pain or palpable soft tissue mass. Development of pathologic fractures is common at initial presentation. The tumors are commonly clinically aggressive. The diagnosis and management warrant a multidisciplinary approach that includes the primary physician, medical oncologist, surgeon, pathologist and radiologist. Surgical resection is the most common treatment with optional neoadjuvant or adjuvant chemotherapy and/or radiation therapy for high grade or dedifferentiated chondrosarcoma with positive margins.

\subsection{Radiologic Findings}

Radiologic screening of chondrosarcomas involves multimodality characterization which is determined according to the American College of Radiology (ACR) appropriateness criteria [4]. The criteria are based upon a grading system which has the following values: rating system of $1-9$, where 9 is the most appropriate measure. The initial imaging modality of localized bone tumors is plain radiography of the region of interest, rating 9. Computed tomography (CT) with and without contrast, is rating 9 if the lesion cannot be adequately characterized on radiographs. Magnetic resonance imaging (MRI) with and without contrast, is rating 8 if the lesion is indeterminate or a lytic lesion. Technetium-99m (Tc-99m) bone scan, rating 5 if better localization of the lesion is needed. Positron emission tomography (PET), is rating 1 . PET is not part of the initial screening of lesions. It is reserved for staging and evaluating of metastatic lesions.

Chondrosarcomas of the rib are most commonly found at the costochondral junction [1]. Radiographically they may be seen as a lytic lesion with endosteal scalloping, cortical thinning or thickening and irregular margins. Calcifications of the mass commonly have a ring and arcs configuration, however, they may be punctate as well. Soft tissue extension is relatively common as demonstrated by our patient's case.

Computed tomography (CT) scans provide superior bony characterization of the mass. It allows for multi-planar (axial, sagittal and coronal) and three dimensional reconstruction, if necessary. The degree of bony destruction and matrix mineralization can easily be identified. Non-mineralized components typically will have low attenuation reflecting their high water content while higher grade lesions tend to show a higher attenuation due to increased cellularity. Low grade lesions tend to exhibit widespread calcifications without soft-tissue exten- 
sion while high grade lesions generally have non-calcified areas with soft-tissue extension. CT performed with intravenous contrast may demonstrate a mild peripheral rim and septal enhancement.

Magnetic resonance imaging (MRI) is excellent at characterizing bone marrow involvement, soft tissue extension and components. Chondrosarcoma lesions tend to demonstrate a lobular morphology. Marrow replacement may appear as low to intermediate signal intensity on T1 weighted images. Non-mineralized components may have high signal intensity on T2 weighted images due to their high water content, while mineralized matrices have low signal intensity with all MR pulse sequences.

Positron emission tomography combined with computed tomography (PET/CT) provides a functional information of the tumor with analysis of active glucose consumption. The higher the metabolic activity the higher the glucose consumption and hence the PET/CT signal. In general, malignant cells have higher metabolic rate relative to the normal soft tissue background. The active lesions will display a high signal which is indicative of active disease.

\subsection{Pathologic Findings}

Chondrosarcoma has chondromyxoid matrix with nuclear pleomorphism, mitosis and necrosis [5]. Permeation of medullary and cortical bone and soft tissue invasion are indicative of malignancy. In addition, the prognosis of chondrosarcoma depends on tumor grade. Grade I is the best prognostic factor without evidence of metastases; Grade II and III are with evidence of metastatic disease with Grade III having the worst outcomes [6]. Chondrosarcomas can originate centrally from the bone or peripherally within the cartilage cap. Central chondrosarcomas are somewhat genetically different from peripheral chondrosarcomas. Central lesions are usually diploid and peripheral lesions are aneuploidy. However, both lesions share the same histology. Other rare chondrosarcoma subtypes include dedifferentiated, mesenchymal and clear cell chondrosarcoma. The dedifferentiated subtype is associated with a very low survival rate due to often distant metastatic involvement at time of initial diagnosis. Mesenchymal chondrosarcomas are usually bi-morphic consisting of low grade cartilaginous cells and hyper cellular small, uniform and undifferentiated cells that resemble Ewing's sarcoma cells. Clear cell chondrosarcomas generally have a good prognosis with complete surgical eradication. Some chondrosarcomas can arise from enchondroma, osteochondroma or extra skeletal myxoid subtypes [6].

\section{Conclusion}

Treatment included primary surgical resection with histology consistent with small, low grade variant chondrosarcoma with low metastatic potential and clear margins. The decision was made not to pursue adjuvant chemotherapy or radiation therapy. Based on the low risk of local recurrence National Comprehensive Cancer Network (NCCN) guidelines recommend surveillance imaging evalua- 
tion of the site with chest CT every 6 months for the first 2 - 3 years followed by yearly follow-up chest CT for a total of 5 - 10 years for this patient.

\section{Conflicts of Interest}

The authors declare no conflicts of interest regarding the publication of this paper.

\section{References}

[1] Murphey, M.D., et al. (2003) From the Archives of the AFIP: Imaging of Primary Chondrosarcoma: Radiologic-Pathologic Correlation. Radio Graphics, 23, 1245-1278. https://doi.org/10.1148/rg.235035134

[2] Ollivier, L., Vanel, D. and Leclere, J. (2003) Imaging of Chondrosarcomas. Cancer Imaging, 4, 36-38. https://doi.org/10.1102/1470-7330.2003.0022

[3] Varma, D.G., Ayala, A.G., Carrasco, C.H., Guo, S.Q., Kumar, R. and Edeiken, J. (1992) Chondrosarcoma: MR Imaging with Pathologic Correlation. Radio Graphics, 12, 687-704.

[4] Kransdorf, M.J., et al. (2018) ACR Appropriateness Criteria Soft-Tissue Masses. Journal of the American College of Radiology, 15, S189-S197. https://doi.org/10.1016/j.jacr.2018.03.012

[5] Brien, E.W., Mirra, J.M. and Luck Jr., J.V. (1999) Benign and Malignant Cartilage Tumors of Bone and Joint: Their Anatomic and Theoretical Basis with an Emphasis on Radiology, Pathology and Clinical Biology. II. Juxtacortical Cartilage Tumors. Skeletal Radiology, 28, 1-20. https://doi.org/10.1007/s002560050466

[6] Rozeman, L.B., Cleton-Jansen, A.M. and Hogendoorn, P.C. (2006) Pathology of Primary Malignant Bone and Cartilage Tumors. International Orthopedics, 30, 437-444. https://doi.org/10.1007/s00264-006-0212-x 